\title{
3D micro-computed tomography of trabecular and cortical bone architecture with application to a rat model of immobilisation osteoporosis
}

\author{
Laib, A ; Barou, O ; Vico, L ; Lafage-Proust, M H ; Alexandre, C ; Rügsegger, P
}

\begin{abstract}
Bone mass and microarchitecture are the main determinants of bone strength. Three-dimensional micro-computed tomogrpahy has the potential to examine complete bones of small laboratory animals with very high resolution in a non-invasive way. In the presented work, the proximal part of the tibiae of hindlimb unloaded and control rats were measured with 3D MicroCT, and the secondary spongiosa of the scanned region was evaluated using direct evaluation techniques that do not require model assumptions. For determination of the complete bone status, the cortex of the tibiae was evaluated and characterised by its thickness. It is shown that with the proposed anatomically conforming volume of interest (VOI), up to an eight-fold volume increase can be evaluated compared to cubic or spherical VOIs. A pronounced trabecular bone loss of $-50 \%$ is seen after 23 days of tail suspension. With the new evaluation techniques, it is shown that most of this bone loss is caused by the thinning of trabeculae, and to a lesser extent by a decrease in their number. What changes most radically is the structure type: the remaining bone is more rod-like than the control group's bone. Cortical bone decreases less than trabecular bone, with only $-18 \%$ after 23 days
\end{abstract}

DOI: https://doi.org/10.1007/bf02347054

Posted at the Zurich Open Repository and Archive, University of Zurich

ZORA URL: https://doi.org/10.5167/uzh-156295

Journal Article

Published Version

Originally published at:

Laib, A; Barou, O; Vico, L; Lafage-Proust, M H; Alexandre, C; Rügsegger, P (2000). 3D micro-computed tomography of trabecular and cortical bone architecture with application to a rat model of immobilisation osteoporosis. Medical Biological Engineering Computing, 38(3):326-332.

DOI: https://doi.org/10.1007/bf02347054 


\title{
3D micro-computed tomography of trabecular and cortical bone architecture with application to a rat model of immobilisation osteoporosis
}

\author{
A. Laib ${ }^{1}$ \\ O. Barou $^{2}$ \\ L. Vico ${ }^{2}$ \\ M. H. Lafage-Proust ${ }^{2}$ \\ C. Alexandre 2 \\ P. Rügsegger ${ }^{1}$ \\ ${ }^{1}$ Institute for Biomedical Engineering, University of Zürich and Swiss Federal Institute of Technology \\ (ETH), Zürich, Switzerland \\ ${ }^{2}$ Laboratory of Bone Biology and Biochemistry, Saint-Etienne University, Saint-Etienne, France
}

\begin{abstract}
Bone mass and microarchitecture are the main determinants of bone strength. Three-dimensional micro-computed tomography has the potential to examine complete bones of small laboratory animals with very high resolution in a non-invasive way. In the presented work, the proximal part of the tibiae of hindlimb unloaded and control rats were measured with $3 D$ MicroCT, and the secondary spongiosa of the scanned region was evaluated using direct evaluation techniques that do not require model assumptions. For determination of the complete bone status, the cortex of the tibiae was evaluated and characterised by its thickness. It is shown that with the proposed anatomically conforming volume of interest (VOI), up to an eight-fold volume increase can be evaluated compared to cubic or spherical VOIs. A pronounced trabecular bone loss of $-50 \%$ is seen after 23 days of tail suspension. With the new evaluation techniques, it is shown that most of this bone loss is caused by the thinning of trabeculae, and to a lesser extent by a decrease in their number. What changes most radically is the structure type: the remaining bone is more rod-like than the control group's bone. Cortical bone decreases less than trabecular bone, with only $-18 \%$ after 23 days.
\end{abstract}

Keywords-Computed tomography (CT), Micro CT, Bone microarchitecture, Rat tibia, Osteoporosis, Morphology

Med. Biol. Eng. Comput., 2000, 38, 326-332

\section{Introduction}

MASS AND architecture are the prime factors influencing the mechanical properties of bone (KLEEREKOPER et al., 1985; MAJUMDAR et al., 1996; PARFITT, 1992; RÜGSEGGER, 1996a, VAN RIETBERGEN et al., 1998). There are currently medications available that increase bone mass in osteoporotic patients, but bone strength is not increased and fracture risk is not lowered as much as expected by the gain in bone density (RIGGS et al., 1990). This suggests that the complex microarchitecture has an important influence on the mechanical competence of bone. In a study with cancellous bone samples from various sites of the human skeleton it was found (ULRICH et al., 1999) that $52 \%$ to $67 \%$ of the elastic modulus is explained by bone density. Including structural indices such as trabecular number, separation and degree of anisotropy, the predictive power is increased to $80-92 \%$. These findings are typical for samples of cancellous bone (GOULET et al., 1994; TURNER et al., 1988).

Correspondence should be addressed to Dr A. Laib e-mail: laib@mrsc.ucsf.edu

First received 31 August 1999 and in final form 13 January 2000

(C) IFMBE: 2000
However, the distribution of cancellous bone is heterogeneous and the question arises as to what extent an evaluated bone sample represents the general trend in the skeleton of the subject, or - more modestly - in a specific bone of a subject. The problem is well known from 2D histomorphometry, where several fields of view are analysed to ease this problem (BIRKENHÄGER-FRENKEL et al., 1988; SNYDER et al., 1993).

Using three-dimensional imaging techniques such as microcomputed tomography, synchrotron CT, micro-magnetic resonance imaging, and serial sectioning (BONSE et al., 1994; FELDKAMP, et al., 1989; HIPP et al., 1996; KINNEY and NICHOLS, 1992; ODGAARD, 1997; RÜGSEGGER et al., 1996b), complete information on the microarchitecture has become available. For these measuring techniques the selection of a representative volume of interest (VOI) is a non-trivial task. It is especially crucial in time-serial examinations where the outcome of a study might well be influenced by the ability to follow a well-defined VOI.

In this work we describe the examination of the proximal part of the rat tibia. The rat is the most widely used animal model of osteoporosis, with ovariectomised rats generally accepted for screening different therapeutic agents and for studying basic effects of bone modelling and remodelling (MOSEKILDE, 1995). The technique proposed in this work 
can also be applied to other animal models such as the mouse (for tibia, femur and vertebrae), or to bone biopsies from larger animals such as sheep or dogs. In this study a model of partial immobilisation of the rat, which unloads the hindlimbs, was used. A three-dimensional conforming volume of interest including the complete secondary spongiosa of the scanned region is used, and structural indices are evaluated with the help of model-independent direct 3D techniques. This is in contrast to traditional procedures where a cubic or spherical VOI (KAPADIA et al., 1998; KINNEY et al., 1998) is used and model-based structural indices are calculated.

Cortical bone was evaluated to give a comprehensive evaluation of the complete bone. To characterise the cortex we analysed its average thickness. Cortical density and moment of inertia are other parameters available from CT images, but are not considered in this work since the main emphasis is on structural information.

To document the behaviour of the proposed evaluation technique it was applied to a controlled experiment to study immobilisation-induced osteoporosis in the tail-suspended rat model.

\section{Materials and methods}

\subsection{MicroCT measurements}

The proximal part of the intact tibiae of rats were measured with a desk-top MicroCT (RÜGSEGGER et al., 1996b], commercially available as $\mu \mathrm{CT} 20^{*}$. It operates with a fan beam originating from a $10 \mu \mathrm{m}$ focal-spot $\mathrm{X}$-ray tube. The photons are detected in a 1024 linear diode array, and the projection data are computer-reconstructed into a $1024 \times 1024$ or $512 \times 512$ matrix. 3D information is obtained by stacking successively measured slices on top of each other. No sample preparation is required, and since MicroCT examinations are non-destructive the bones remain available for other examination techniques afterwards. The chosen voxel size was $24 \mu \mathrm{m}$ in all three spatial dimensions. For each sample, 240 slices were measured, covering a total of $5.76 \mathrm{~mm}$ of the diaphysis and metaphysis. A 3D visualisation of a complete measurement is shown in Fig. 1.

\subsection{Animals}

Eighteen 14-week-old male Wistar rats were acclimatised for one week with standard conditions of temperature $(23 \pm 1 \mathrm{C})$ and light-controlled environment ( $12 \mathrm{~h}$ light-dark cycles). They were fed with standard rodent chow and water ad libitum. Then they were randomly divided into two groups, tail-suspended (S) and control (C). Rats were suspended without anesthesia according to a standard procedure previously described by MOREY (1979) using specially designed cages allowing the animals to walk on their forelimbs. The suspension angle was $30^{\circ}$, so that approximately $50 \%$ of the body weight is loaded onto the forelimbs. Three animals of each group were sacrificed at days 7,13 and 23 with anaesthesic overdose (Nesdonal $0.1 \mathrm{mg} \mathrm{kg}^{-1}$ ). Tibiae were immediately excised and fixed in ice-cold, phosphate-buffered formalin $(\mathrm{pH} 7.4)$ and then placed in $70 \%$ alcohol. The procedure for the care, experimental protocol and killing of the study animals was in accordance with the European Community standards on the care and use of laboratory animals (Ministère de l'Agriculture, France, authorization no. 04827).

\footnotetext{
*Scanco Medical Bassersdorf, Switzerland
}

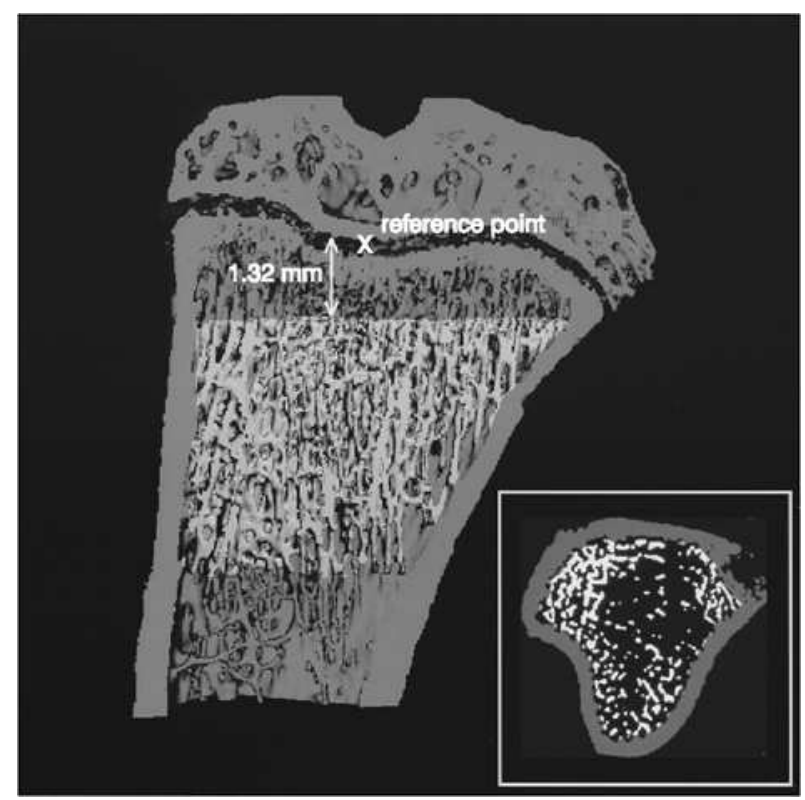

Fig. 1 Cut through the $3 D$ image of a MicroCT measurement of a control rat. The conforming VOI is highlighted. The reference point at the end of the epiphysis is shown as a cross, from which the conforming region starts at a fixed distance of $1.32 \mathrm{~mm}$. The inset show's a transaxial cut through the bone with the VOI highlighted

\subsection{Determination of trabecular VOI}

An outer bone contour was produced automatically slice-byslice, following a rough contour that had been drawn by hand in the starting slice. Contour finding is based on a steepest gradient determination of the gray-scale levels of background (alcohol in this case) versus tibia cortex, with side conditions of maximum curvature and connectedness of isolated, single parts to ensure robustness against pixel noise (MüNCH, 1991).

To analyse the trabecular part of the tibiae, the compact part of the bone was masked in the following way: with a threedimensional box-shaped low-pass filter (support of 13 voxels in each spatial direction) applied to the original gray-scale CT images, an artificial partial volume effect was created, which blurred out the individual trabeculae, but left the dense compact shell intact. The cortex mask was then extracted with a simple thresholding operation with a fixed threshold of $15.0 \%$ of the maximal gray-scale value. Isolated speckles remaining on the inside of the cortex mask were removed with a slicewise component labelling and extraction of the main component, i.e. the cortex. To determine the region of interest in the axial direction, a reference point was found visually (Fig. 1) and then the region of interest in our fixed distance of 55 slices $(=1.32 \mathrm{~mm}$ ) with a length of $4.32 \mathrm{~mm}$ (=180 slices) was chosen automatically. The distance of $1.32 \mathrm{~mm}$ was chosen such that no primary spongiosa was included in the trabecular VOI in any of the samples.

\subsection{Segmentation and 3D morphometric evaluation of trabecular $\mathrm{VOI}$}

The gray-value images were segmented using a low-pass filter to remove noise and a fixed threshold to extract the mineralised bone phase (Gaussian filter with $\mathrm{s}=0.7$ voxels and threshold $10.5 \%$ of maximal gray-scale value).

Bone volume BV and bone surface BS were calculated using a tetrahedron meshing technique generated with the Marching Cubes method (LORENSEN and CLINE, 1987). Total volume TV was calculated from the volume of the conforming VOI. Mean 
trabecular number, mean trabecular thickness, and mean trabecular separation were calculated using newly developed direct techniques based on the distance transformation (HILDEBRAND and RÜGSEGGER, 1997a, HILDEBRAND et al., 1999) of the binary object, and were denoted Tb. ${ }^{*}, \mathrm{~Tb}^{*} \mathrm{Th}^{*}$ and $\mathrm{Tb} . \mathrm{Sp}^{*}$, respectively (asterisks are used to distinguish them from the indices Tb.N, Tb.Th, Tb.Sp calculated according to PARFITT et al., (1983). Even though the discrete representation of the structure with $24 \mu \mathrm{m}$ voxels is relatively coarse in comparison to the thickness of trabeculae, the very large number of voxels over which the mean values of thickness and separation are calculated (in this study of the order of 3000000 voxels per sample) still assures reliable values for these indices. In a previous study (PEYRIN et al., 1998) measurements of bone samples from human vertebrae made with our MicroCT were compared with those made using a $6.6 \mu \mathrm{m}$ Synchrotron CT. The correlations between the two procedures were above 0.93 for $\mathrm{BV} / \mathrm{TV}, \mathrm{Tb} . \mathrm{N}^{*}, \mathrm{~Tb} . \mathrm{Sp}^{*}$ and Tb. Th* .

An estimation of the plate-rod characteristic of the structure is achieved using the Structure Model Index, SMI (HILDEBRAND and RÜESEGGAR, 1997b, HILDEBRAND et al., $1997 c$ ]. It is calculated by a differential analysis of a triangulated surface of a structure and is defined as

$$
\mathrm{SMI}=6 \cdot \frac{B V \cdot \frac{\mathrm{d} B S}{\mathrm{~d} r}}{B S^{2}}
$$

where $\mathrm{d} B S / \mathrm{d} r$ is the surface area derivative with respect to a linear measure $r$, corresponding to the half-thickness or the radius assumed constant over the entire structure. This derivative is estimated by a simulated thickening of the structure by translating the triangulated surface a small extent in its normal direction and dividing the associated change of surface area with the length of the extent. For an ideal plate and rod structure the SMI value is 0 and 3, respectively. For a structure with both plates and rods of equal thickness the value is between 0 and 3 , depending on the volume ratio between rods and plates.

The geometrical degree of anisotropy, DA, is usually defined as the ratio between the maximum and the minimum radius of the mean intercept length (MIL) ellipsoid (HARRIGAN and MANN, 1984; WHITEHOUSE, 1974). For our irregular volumes, however, we did not use test lines through the volume, but calculated a direction distribution of the projected triangulated surfaces by calculating the scalar product of the area weighted normal vector of each surface triangle with the discretised directions of the direction distribution. This distribution corresponds to the inverse of the usually computed intercept length distribution. The 'quasi MIL' ellipsoid is then calculated by fitting the inverse directional projected surface distribution to an ellipsoid using a least squares fit. The advantage of not using test lines is the avoidance of sampling problems inherent in the MIL method, proven in SIMMONS and HIPP (1997) to lead to variations of as much as $52 \%$ in the MIL indices.

The artificial surfaces of the 'cut' trabeculae created by the boundaries of the VOI were suppressed in the calculation of all the surface-related indices such as BS, SMI and DA, so that the inherent anisotropy of the conforming VOI induces no bias into the results.

Connectivity density, (Conn.D.) was calculated using the Euler method of ODGAARD and GUNDERSEN (1993). The axial borders (flat top and bottom) were treated with the boundary correction as described in ODGAARD and GUNDERSEN (1993) for unknown continuation of objects. To deal with the problem of the conforming transaxial boundary, we placed a one-voxel shell around the VOI again before calculating Conn.D., because we knew that originally all the trabeculae at the boundary were going into the cortex and were thus connected to each other.

\subsection{Determination and thickness evaluation of cortical VOI}

Although cancellous bone is the main target when documenting changes due to disease and treatment since its turnover is eight times faster than that of cortical bone, cortical bone plays an equally important role with regard to mechanical stability. In cases of severe osteoporosis with very weak cancellous bone, load transfer occurs primarily via the cortex. Since in our measurement the whole proximal end of the tibia is measured, it is possible to evaluate not only cancellous bone, but also cortical bone. To analyse the cortex, the original images were low-pass filtered with a Gaussian filter $(s=3.0)$, and subsequently a threshold was applied of $10.0 \%$. The axial position and extent was identical with the trabecular volume. The trabecular region was masked out and a 3D component labelling of the cortex was additionally performed to extract the main connected component only. Then the cortex was evaluated using the direct distance transformation method to calculate its thickness, i.e. spheres were 'blown up' in the cortex and the mean of the diameter of these spheres was calculated.

\section{Results}

The success of the conformity of the trabecular VOI is shown in Figs 1 and 2. The complete trabecular part of the chosen axial extent is analysed, while none of the cortical shell intrudes. The VOI should be as large as possible to give representative results. Limits are given in the proximal direction by the primary spongiosa, and in the distal direction by the fact that spongiosa 'peters out' and that no additional information is gained by the inclusion of an almost empty volume in both control and suspension rats. To decide which length of VOI might be meaning ful we analysed two rats, a 7-day control rat and a 23-day suspended rat, at the beginning of the study. In Fig. 3 the relative difference of $\mathrm{BV} / \mathrm{TV}$ of the two rats is plotted against the axial dimension of the conforming VOI. The curve shows large fluctuations at the beginning when very

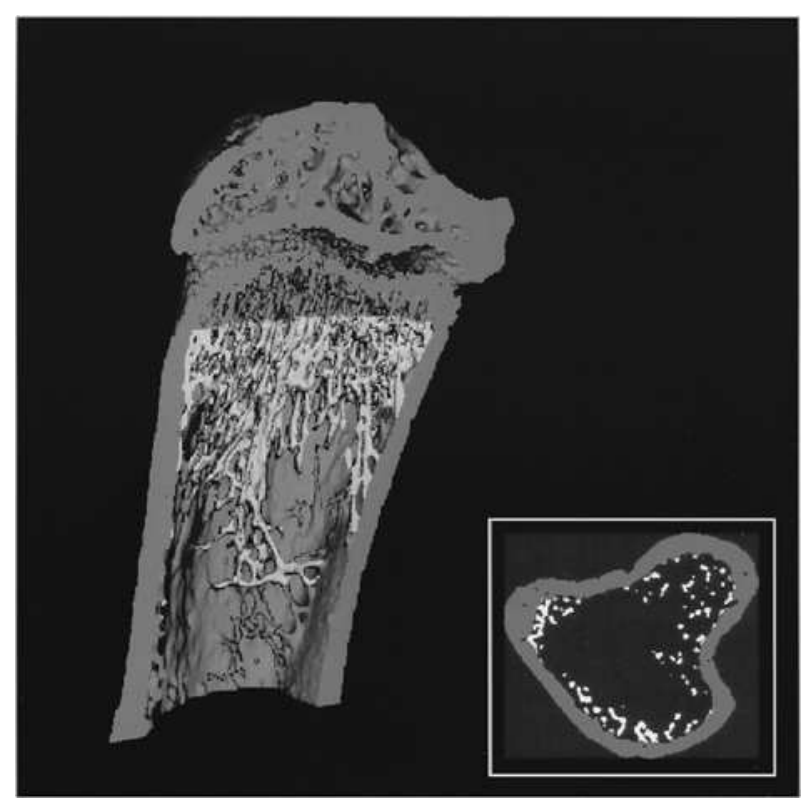

Fig. 2 Same as Fig. 1 but for a 23-day suspended rat. The volume of interest is highlighted, the inset shows a transaxial cut 


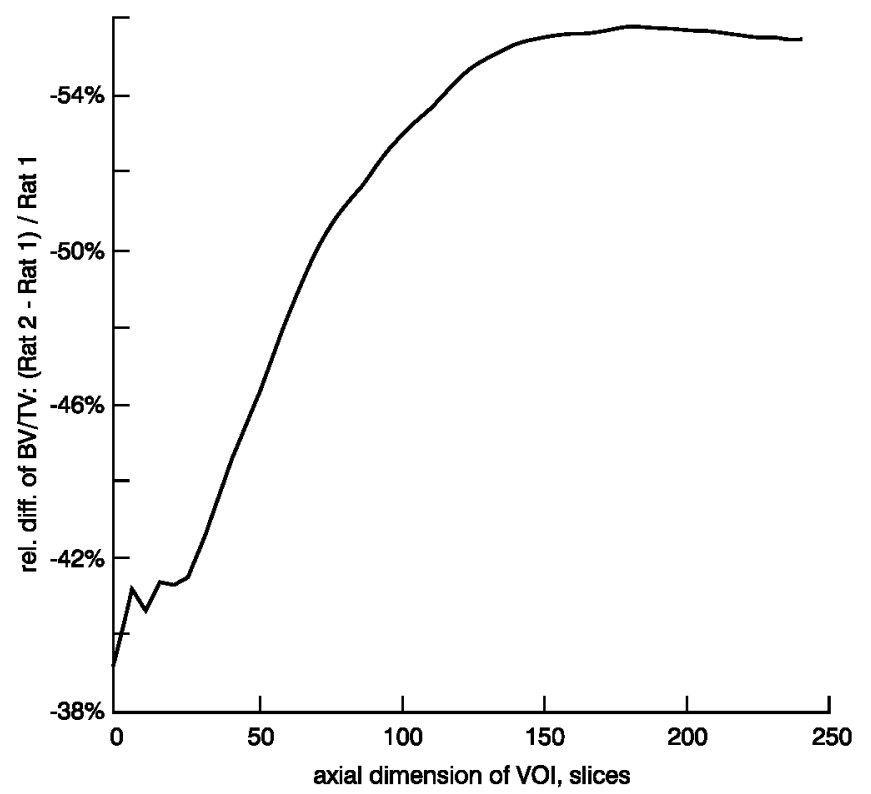

Fig. 3 Choice of the axial extent of the VOI. The axial length of the VOI is then selected such that the relative amount of analved bone is optimal with respect to the difference between control and suspended rats. Rat 1 is a 7-dav control rat. Rat 2 a 23day suspended rat. The curve show's a maximum around 180 slices

small VOIs are analysed, then a gradual rise (the control rat still has dense spongiosa up to the axial position while the suspended rat already has 'holes') and a flat maximum around an axial length of VOI of 180 slices, before the curve falls again as control rat's spongiosa 'peters out'. The complete series of rats was then evaluated using 180 slices. 'Exploded' views of the chosen trabecular and cortical VOI of the 7-day control and 23-day suspended rat are shown in Figs 4 and 5, with the spongiosa artificially lifted out of the cortex for illustration purposes.

In Table 1 the group averages with standard deviations (SD) are given for all the indices. The relative day-matched differences are given in Table 2 (no $p$-values given for such small group sizes of only three).

Clearly visible is the progression of trabecular bone loss with prolonged tail suspension: while the relative difference of $\mathrm{BV} / \mathrm{TV}$ is $-16 \%$ in the 7-day suspended group as against the 7-day control group, it is already $-23 \%$ lower after 13 days, and $-50 \%$ lower after 23 days. BS/BV shows an increase of $+14 \%$, then $+18 \%$ and $+10 \%$ after 23 days.

Cancellous bone structure is usually analysed using procedures originally developed for 2D histomorphometry, where

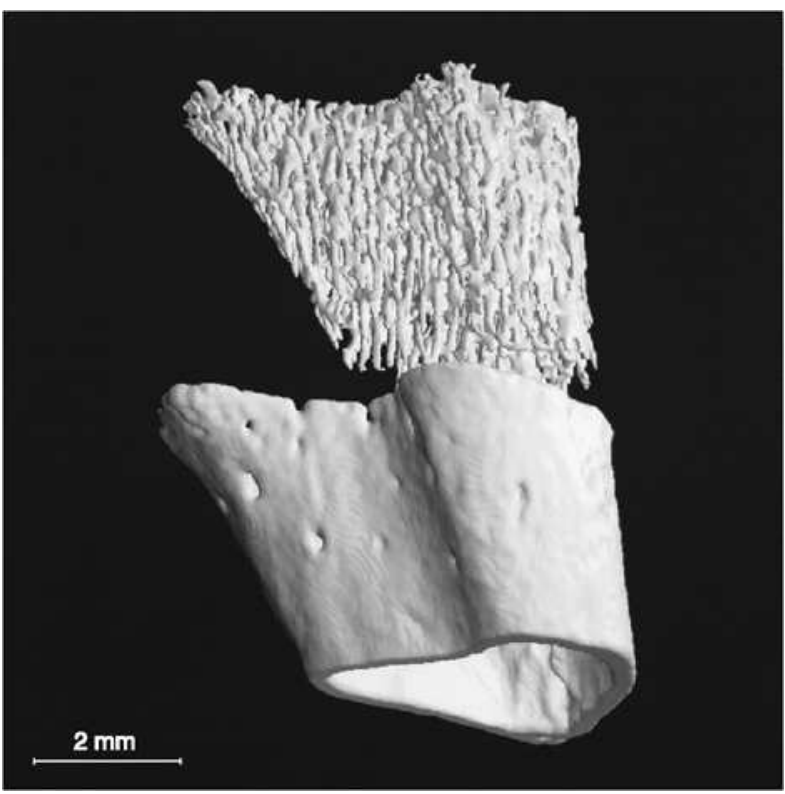

Fig. 4 'Exploded' view of cancellous and cortical bone of a 7-day control rat. Relative bone volume is normal at $22 \%$, the structure model index is 2.2 corresponding to a mixed platelike rod-like structure, cortical thichness is $0.280 \mathrm{~mm}$

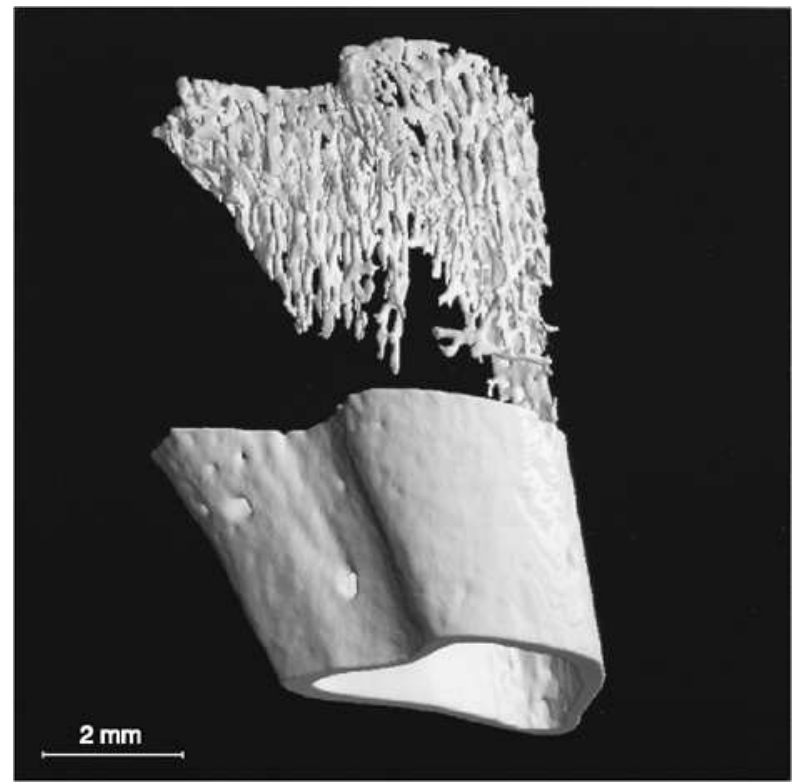

Fig. 5 Same as Fig. 4 for a 23-day suspended rat. BV/TV is $9 \%$, SMI 2.93 (cylindrical rods), C.Th $0.267 \mathrm{~mm}$

Table 1 Mean values and standard deviations (SD) of the structural indices at day 7, 13 and 23, for tail suspended rats and control rats

\begin{tabular}{|c|c|c|c|c|c|c|}
\hline \multirow[b]{2}{*}{ Structural index } & \multicolumn{6}{|c|}{ Group } \\
\hline & $\begin{array}{c}\text { Day } 7 \\
\text { Control } \\
\text { Mean } \pm \text { SD }\end{array}$ & $\begin{array}{c}\text { Day } 7 \\
\text { Suspended } \\
\text { Mean } \pm \text { SD }\end{array}$ & $\begin{array}{c}\text { Day } 13 \\
\text { Control } \\
\text { Mean } \pm \text { SD }\end{array}$ & $\begin{array}{c}\text { Day } 13 \\
\text { Suspended } \\
\text { Mean } \pm \text { SD }\end{array}$ & $\begin{array}{c}\text { Day } 23 \\
\text { Control } \\
\text { Mean } \pm \text { SD }\end{array}$ & $\begin{array}{c}\text { Day } 23 \\
\text { Suspended } \\
\text { Mean } \pm \text { SD }\end{array}$ \\
\hline $\mathrm{BV} / \mathrm{TV}$ [1] & $0.19 \pm 0.04$ & $0.16 \pm 0.04$ & $0.22 \pm 0.02$ & $0.17 \pm 0.02$ & $0.18 \pm 0.02$ & $0.09 \pm 0.01$ \\
\hline $\mathrm{BS} / \mathrm{BV}, \mathrm{mm}^{-1}$ & $24.4 \pm 1.7$ & $27.7 \pm 1.9$ & $22.8 \pm 0.6$ & $27.0 \pm 1.7$ & $24.8 \pm 1.6$ & $27.2 \pm 3.1$ \\
\hline Tb. $\mathrm{N}^{*}, \mathrm{~mm}^{-1}$ & $2.45 \pm 0.40$ & $2.86 \pm 0.86$ & $2.68 \pm 0.19$ & $2.86 \pm 0.09$ & $2.01 \pm 0.09$ & $1.51 \pm 0.39$ \\
\hline Tb.Th* ${ }^{*}, \mu \mathrm{m}$ & $83 \pm 5$ & $77 \pm 3$ & $88 \pm 3$ & $79 \pm 4$ & $81 \pm 1$ & $72 \pm 6$ \\
\hline Tb.Sp* ${ }^{*}, \mu \mathrm{mj}$ & $438 \pm 77$ & $383 \pm 136$ & $388 \pm 28$ & $352 \pm 13$ & $507 \pm 31$ & $725 \pm 222$ \\
\hline SMI [1] & $2.22 \pm 0.23$ & $2.61 \pm 0.16$ & $2.08 \pm 0.11$ & $2.57 \pm 0.25$ & $2.25 \pm 0.29$ & $2.96 \pm 0.08$ \\
\hline DA [1] & $1.96 \pm 0.07$ & $2.02 \pm 0.16$ & $1.96 \pm 0.04$ & $1.88 \pm 0.03$ & $1.99 \pm 0.04$ & $2.03 \pm 0.18$ \\
\hline Conn.D., $\mathrm{mm}^{-3}$ & $44.0 \pm 6.7$ & $38.2 \pm 11.5$ & $43.6 \pm 3.2$ & $39.0 \pm 0.8$ & $42.2 \pm 6.7$ & $19.0 \pm 2.1$ \\
\hline C.Th, $\mu \mathrm{m}$ & $319 \pm 36$ & $286 \pm 16$ & $355 \pm 5$ & $284 \pm 54$ & $335 \pm 38$ & $276 \pm 19$ \\
\hline
\end{tabular}


Table 2 Mean differences of the structural indices after 7 , 13 and 23 davs of suspended rats (S) and control rats (C) relative to mean values of control groups.

\begin{tabular}{|c|c|c|c|}
\hline Relative changes & $\begin{array}{c}\text { Day } 7 \\
(\mathrm{~S}-\mathrm{C}) / \mathrm{C}\end{array}$ & $\begin{array}{l}\text { Day } 13 \\
(\mathrm{~S}-\mathrm{C}) / \mathrm{C}\end{array}$ & $\begin{array}{l}\text { Day } 23 \\
(\mathrm{~S}-\mathrm{C}) / \mathrm{C}\end{array}$ \\
\hline $\mathrm{BV} / \mathrm{TV}$ [1] & $-16 \%$ & $-23 \%$ & $-50 \%$ \\
\hline $\mathrm{BS} / \mathrm{BV}, \mathrm{mm}^{-1}$ & $+14 \%$ & $+18 \%$ & $+10 \%$ \\
\hline Tb. $\mathrm{N}^{*}, \mathrm{~mm}^{-1}$ & $+17 \%$ & $+7 \%$ & $-25 \%$ \\
\hline $\mathrm{Tb} . \mathrm{Th}^{*}, \mu \mathrm{m}$ & $-7 \%$ & $-10 \%$ & $-11 \%$ \\
\hline $\mathrm{Tb} . \mathrm{Sp}^{*}, \mu \mathrm{m}$ & $-13 \%$ & $-9 \%$ & $+43 \%$ \\
\hline SMI [1] & $+18 \%$ & $+24 \%$ & $+32 \%$ \\
\hline DA [1] & $+3 \%$ & $-4 \%$ & $+2 \%$ \\
\hline Conn.D., $\mathrm{mm}^{-3}$ & $-13 \%$ & $-11 \%$ & $-55 \%$ \\
\hline C.Th, $\mu \mathrm{m}$ & $-10 \%$ & $-20 \%$ & $-18 \%$ \\
\hline
\end{tabular}

$\mathrm{BV} / \mathrm{TV}$; bone volume to total volume.

$\mathrm{BS} / \mathrm{BV}$; bone surface to bone volume.

$\mathrm{Tb} \mathrm{N}^{*}, \mathrm{~Tb} \mathrm{Th}^{*}, \mathrm{~Tb} . \mathrm{Sp}^{*}$; trab. number, thickness, separation (direct method).

SMI; structure model index (0 plate, 3 rod).

DA; degree of anisotropy.

Conn.D; connectivity density.

C.Th; cortical thickness (direct method).

extensions to $3 \mathrm{D}$ require model assumptions. In addition to the direct evaluation techniques described in Section 2.4 we analysed our MicroCT examinations using standard classical procedures, assuming a plate model (PARFITT et al., 1983). The relative differences between tail suspended rats and control rats obtained with these model-dependent procedures are $-11 \%$, $-14 \%$ and $-8 \%$ for $\mathrm{Tb}$.Th, and $-3 \%,-9 \%$ and $-46 \%$ for Tb.N for the 7-, 13-, and 23-day measurements. These results suggest that tail suspension causes thinning of trabeculae and a continuous decrease in their number, as also reported in the literature (LI et al., 1990; MAEDA et al., 1993). This interpretation is not supported by the direct 3D indices given in Table 2. The Structure Model Index, SMI (Fig. 6), shows a change from 2.0 to 2.96 ( 3 being an ideal rod-like structure) with relative differences of $+18 \%,+24 \%$ and $+32 \%$ at day 7 , 13 and 23 . Hence a mixed plate-rod structure is transformed into a pure rod-like structure. Tb. $N^{*}$ increases first (day 7) due to fenestration of plates, the number of elements increases, then (day 13) the formation of rods from plates and the elimination of rods are more or less equal, then (day 23) Tb. $\mathrm{N}^{*}$ decreases due to the elimination of rods. A refinement of this view is given by Conn.D., which remains relatively stable at first, but

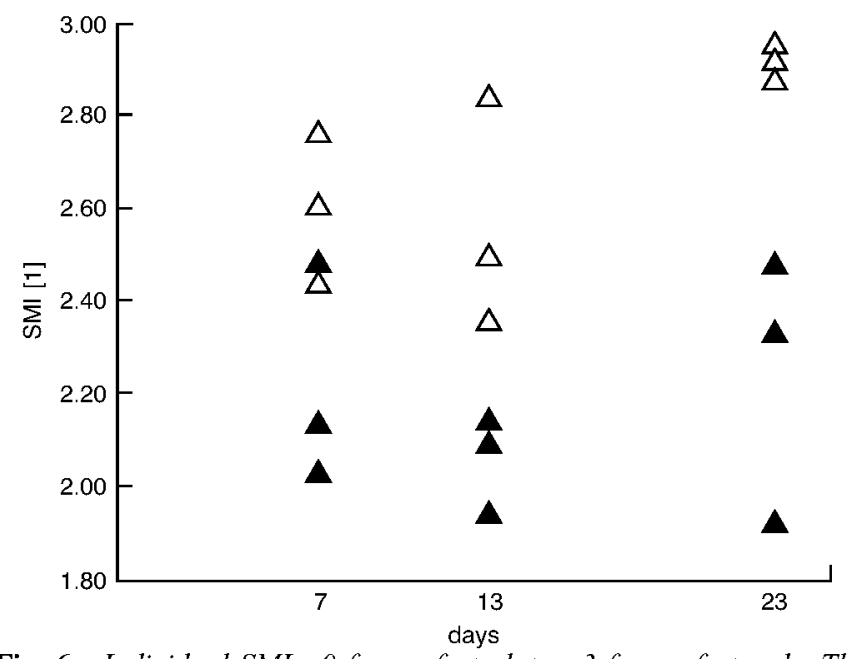

Fig. 6 Individual SMIs. O for perfect plates, 3 for perfect rods. The trend towards rod-like trabeculae is clearly seen with prolonged tail suspension. A control: $\triangle$ suspended later on shows a sharp drop (-55\% after 23 days of tail suspension).

The cortex shows a decline of $-10 \%,-20 \%$ and $-18 \%$ in its thickness after 7,13 and 23 days. It seems that trabecular and cortical bone loss become uncoupled as the time of tail suspension increases, when the $50 \%$ trabecular bone loss is compared to the $18 \%$ cortical bone loss.

\section{Discussion}

In the work presented, rats with the hind limb unloaded were examined using MicroCT measurements. The proximal tibiae of male Wistar rats were imaged in three dimensions and a maximum trabecular volume of interest was chosen in the secondary spongiosa with conforming contours and mask-off of the cortex, which in turn was evaluated separately. The 3D CT images were processed with different morphometric evaluation techniques. Traditional methods, which assume a plate model for trabecular bone, and recently introduced direct methods, which assess bone independently of a model assumption, and a method which quantifies the structure type of the measured bone were applied. Artificially created boundaries were suppressed and accounted for in the evaluation steps. The cortex was assessed for its thickness with direct methods.

The trabecular VOI consisted of the complete secondary spongiosa of the proximal metaphysis of the tibia, which was determined with only two minor user interactions: the rough starting contour for automatic contour finding, and an indication of the anatomical landmark to determine the axial position. With the proposed conforming methods, the axial dimension can be freely chosen, in contrast to cubic or spherical VOIs where the axial dimension is coupled to the other two transverse dimensions. When compared to box-shaped VOIs or spherical VOIs that have to fit inside the trabecular region, a much larger volume is evaluated with our conforming method (roughly four to eight times larger, $\sim 47 \mathrm{~mm}^{3}$ conforming; $\sim 12 \mathrm{~mm}^{3}$ cubic or $\sim 6 \mathrm{~mm}^{3}$ spherical) and the considerable variability of results brought about by placing a VOI by hand is avoided.

The conforming VOI has an additional potential: it allows one to analyse regional differences. Using peeling operations it is possible to evaluate the core of the trabecular region where bigger changes might be expected; or, alternatively, changes in the ring-shaped region closest to the cortex might be of interest. This separation requires no additional user interactions since it is based on automatic contouring, and thus additional information about the homogeneity of bone is available 'free of charge'. (Data for different VOIs not shown here, since the small group size prevents meaningful statistics and discussion of differential VOIs.)

MicroCT is a very efficient tool to detect bone loss in immobilisation osteoporosis. However, the plate model assumption used in standard evaluation gives strong bias of the structural indices when the model assumption is increasingly violated by rodification of bone. This trend of conversion from plates to broad oval rods to cylindrical rods was quantified on this set of rat bones using the SMI. While the standard plate model assuming indices Tb.Th and Tb.N both show a gradual decline with prolonged hind limb unloading (which agrees with results from conventional 2D histomorphometry (LI et al., 1990), which also assumes a plate model for bone), this is not the case with the direct, model-assumptionfree indices: bone loss is primarily caused by the thinning of trabeculae and not by a decrease in the number. Only after 23 days of unloading does $\mathrm{Tb} . \mathrm{N}^{*}$ drop. This is also reflected in the connectivity density, which shows, after 23 days, a pronounced decline of $55 \%$. 
The number of rats per group was very small in our study and restricts extensive statistical analysis. Nevertheless, the additional information gained with new morphometric techniques is illustrated. It is worth noting that direct Tb.N* and Conn.D. are related to each other. However, there is an important difference: Tb. $\mathrm{N}^{*}$ is a real metric index, namely the inverse of the mean distance between the midlines of trabeculae, and is determined by the diameter of the largest sphere fitting inside them. Conn.D. relates to the number of connections found in the VOI irrespective of their distance from each other. It is thus possible that, for example, the disappearance of a horizontal rod has no effect on $\mathrm{Tb} . \mathrm{N}^{*}$ because the distance spheres are bounded by vertical rods at that place, but Conn.D., on the other hand, is reduced.

The advance in three-dimensional imaging techniques allows study the organisation of trabecular and cortical architecture in great detail. Novel methods give valuable new insight into the remodelling processes involved in osteoporosis. The considerable individual differences found within the groups underline the importance of in vivo 3D MicroCT (KOHLBRENNER et al., 1999), where each animal serves as its own control and changes in the microstructure can be followed directly in the same animal. The full potential of the proposed evaluation techniques will be realized when these new tools are available.

Acknowledgments-This work was supported in part by grant 3145811.95 from the Swiss National Science Foundation and European Space Agency ESA (ESTEC grant 12291).

\section{References}

Birkenhäger-Frenkel, D. H., Coupron, P., Hüpscher, E. A., Clermonts, E., Coutinho, M. F., Schmitz, P. I. M., and MeUNIER, P. J. (1988): 'Age-related changes in cancellous bone structure, a two-dimensional study in the transiliac iliac crest biopsy sites', Bone Min., 4, pp. 197-216

Bonse, U., Busch, F., Günnewig, O., Beckmann, F., Pahl, R., Delling, G., HAHN, M., and GRAEFF, W. (1994): '3D computed Xray tomography of human cancellous bone at $8 \mu \mathrm{m}$ spatial and $10^{-4}$ energy resolution,' Bone Min., 25, pp. 25-38

FeldKamp, L. A., Goldstein, S. A., Parfitt, A. M., Jesion, G., and KLEEREKOPER, M. (1989): 'The direct examination of three-dimensional bone architecture in vitro by computed tomography', J. Bone Min. Res., 4, pp. 3-11

Goulet, R. W., Goldstein, S. A., Ciarelli, M. J., Kuhn, J. L., Brown, M. B., and FeldKAMP, L.A. (1994): 'The relationship between the structural and orthogonal compressive properties of trabecular bone', J. Biomech., 27, pp. 375-389

HARRIGAN, T. P., and MANN, R. W. (1984): 'Characterization of microstructural anisotropy in orthotropic materials using a second rank tensor', J. Mater: Sci., 19, pp. 761-767

HILDEBRAND, T., and RÜGSEGGER, P. (1997a): 'A new method for the model independent assessment of thickness in three-dimensional images', J. Microsc., 185, pp. 67-75

Hildebrand, T., and RüGSEGGer, P. (1997b): 'Quantification of bone microarchitecture with the structure model index', Comput. Meth. Biomech. Biomed. Eng., 1, pp. 15-23

Hildebrand, T., Laib, A., Ulrich, D., Kohlbrenner, A., and RÜGSEGGER, P. (1997c): 'Bone structure as revealed by microtomography', Proc. SPIE, 3149, pp. 34-43

HILDEbrand, T., LAIB, A., MÜLlER, R., DEQUekER, J., and RÜGSEGGER, P. (1999): 'Direct 3D morphometric analysis of human cancellous bone: microstructural data from spine, femur, iliac crest and calcaneus', J. Bone Miner: Res., 14, 1167-1174

Hipp, J. A., JANSUJWICZ, A., Simmons, C. A., and SNYDER, B. (1996): 'Trabecular bone morphology from micro-magnetic resonance imaging', J. Bone. Miner: Res., 11, pp. 286-297

Kapadia, R. D., Stroup, G. B., Badger, A. M., Koller, B., Levin, J. M., COATnEY, R. W., DODDS, R. A., Liang, X., LARK, M. W., and
Gowen, M. (1998): 'Applications of micro-CT and MR microscopy to study pre-clinical models of osteoporosis and osteoarthritis', Technol. Health Care, 6, pp. 361-372

Kleerekoper, M., Villanueva, A. R., Stanciu, J., RaO, D. S., and PARFITT, A. M. (1985): 'The role of three-dimensional trabecular microstructure in the pathogenesis of vertebral compression fractures', Calcif. Tissue Int., 37, pp. 594-597

KinNEY, J. H., and Nichols, M. C. (1992): 'X-ray tomographic microscopy (XTM) using synchrotron radiation', Annu. Rev: Mater: Sci., 22, pp. 121-152

KinNEY, J. H., RYABY, J. T., HAUPT, D. L., and LANE, N. E. (1998): 'Three-dimensional in vivo morphometry of trabecular bone in the OVX rat model of osteoporosis', Technol. Health Care, 6, pp. 339350

Kohlbrenner, A., HÄmmerle, S., Laib, A., Koller, B., and RÜGSEGGER, P. (1999): 'Fast 3D multiple fan-beam CT systems', Proc. SPIE, 3772, pp. 44-54

LI, X. J., Jee, W. S. S., Chow, S-Y., and Woodbury, D. M. (1990): 'Adaptation of cancellous bone to aging and immobilization in the rat: a single photon absorptiometry and histomorphometry study', Anat. Rec., 227, pp. 12-24

LORENSEN, W. E., and CLINE, H. E. (1987): 'Marching Cubes: A high resolution 3D surface construction algorithm', Comput. Graphics, 21, pp. $163-169$

Maeda, H., Kimmel, D. B., RAab, D. M., and LANe, N. E. (1993): 'Musculoskeletal recovery following hindlimb immobilization in adult female rats/, Bone, 14, pp. 153-159

Majumdar, S., Newitt, D., Mathur, A., Osman, D., Gies, A., ChIU, E., LotZ, J., KinNEY, J., and GenANT, H. (1996): 'Magnetic resonance imaging of trabecular bone structure in the distal radius: relationship with $\mathrm{x}$-ray tomographic microscopy and biomechanics', Osteoporosis Int., 6, pp. 376-385

MOREY, E. R. (1979): 'Spaceflight and bone turnover correlation with a new model of weightlessness', Bio. Sci., 29, pp. 168-172

MOSEKILDE, L. (1995): 'Asessing bone quality - animal models in preclinical osteoporosis research', Bone, 17, pp. 345S 352S

MÜNCH, B. (1991): '3D-Analyse von Knietomogrammen'. Diss. ETH Zuerich Nr. 9459

OdgaARD, A., and Gundersen, H. J. G. (1993): 'Quantification of connectivity in cancellous bone, with special emphasis on 3D reconstructions', Bone, 14, pp. 173-182

ODGAARD, A. (1997): 'Three-dimensional methods for quantification of cancellous bone architecture', Bone, 20, pp. 315-328

Parfitt, A. M., Mathews, C. H. E., Villanueva, A. R., KleerEKoPER, M., Frame, B., and RAO, D. S. (1983): 'Relationships between surface, volume, and thickness of iliac trabecular bone in aging and in osteoporosis', Calcif. Tissue Int., 72, pp. 1396-1409

PARFITT, A. M. (1992): 'Implications of architecture for the pathogenesis and prevention of vertebral fracture', Bone, 13, pp. S41S47

Peyrin, F., Salome, M., Cloetens, A. M., Laval-Jeantet, A. M., RITMAN, E., and RÜGSEGGER, P. (1998): 'Micro-CT examinations of trabecular bone samples at different resolutions: 14, 7 and 2 micron level', Technol. Health Care., 6, pp. 391-401

Riggs, B. L., Hodgson, S. F., O'fallon, W. M., ChaO, E. Y., Wahner, H. W., Muhs, J. M., Cedel, S. L., and Melton, J. M. (1990): 'Effect of fluoride treatment on the fracture rate in postmenopausal women with osteoporosis', New Engl. J. Med., 332, pp. $802-809$

RÜGSEGGER, P. (1996a): 'Bone density measurement' in Bröll, H., and Dambacher, M. A., (Eds): 'Osteoporosis: A guide to diagnosis and treatment. Rheumatology'. Basel, Karger, 18, pp. 103-116

RÜGSEGger, P., Koller, B., and MÜLLeR, R. (1996b): 'A microtomographic system for the non-destructive evaluation of bone architecture', Calcif. Tissue Int., 58, pp. 24-29

Simmons, C. A. and HiPP, J. A. (1997): 'Method-based differences in the automated analysis of the three-dimensional morphology of trabecular bone', J. Bone Miner: Res., 12, pp. 942-947

SNYDER, B. D., PIAZZA, S., EdWARd, W. T., and Hayes, W. C. (1993): 'Role of trabecular morphology in the etiology of agerelated vertebral fractures', Calcif. Tissue Int., 53, pp. S14-S22

Turner, C. H., Rho, J. Y., Ashman, R. B., and Cown, S. C. (1988): 'The dependence of elastic constants of cancellous bone upon structural density and fabric', Trans. Orthopaed. Res. Soc., 13, pp. 74 
Ulrich, D., Van Rietbergen, B., Laib, A., and RÜgsegger, P. (1999): 'The ability of 3D structural indices to reflect mechanical aspects of trabecular bone', Bone., 25, pp. 55-60

Van rietbergen, B., Majumdar, S., Pistoia, W., Newitt, D. C., Kothari, M., LAIB, A., and RÜGSEgGer, P. (1998): 'Assessment of cancellous bone mechanical properties from micro-FE models based on micro-CT, pQCT and MR images', Technol. Health Care., 6, pp. 413-420

WhiteHousE, W.J. (1974): 'The quantitative morphology of anisotropic trabecular bone', J. Microsc., 101, pp. 153-168

\section{Author's biography}

ANDRES LAIB received his MSc degree in experimental physics in 1994 and his PhD degree in physics in 1999 from the ETH Zürich, Switzerland on the subject of high-resolution PQCT of the human forearm. He currently holds a post-doctorate position at the Magnetic Resonance Science Center at the University of California in San Francisco. His main research interests are in bone structure analysis, computed tomography and magnetic resonance imaging. 